\section{Determination of silencing potency of synthetic and RNase III-generated siRNA using a secreted luciferase assay}

\author{
Jean-Étienne Morlighem, Céline Petit, and George Tzertzinis \\ New England Biolabs, Ipswich, MA, USA \\ BioTechniques 42:599-606 (May 2007) \\ doi 10.2144/000112444
}

\begin{abstract}
RNA interference (RNAi) is an established tool for functional genomics studies that is also showing great potential for medical applications. Currently, one of the main goals in RNAi technology is the design and discovery of potent small interfering RNAs (siRNAs). Using a secreted luciferase from Gaussia princeps (GLuc), we developed a reporter assay, which allows for rapid potency assessment of siRNAs, by measuring luminescence activity in cell culture supernatants. The method was applied in microtiter plate format and validated by comparison to quantitative reverse transcription PCR (RT-PCR) and Western blot analysis. This reporter assay was used to evaluate in HeLa cells the potency of different siRNA mixtures generated by RNase III, or several synthetic siRNAs, all directed against human p53. The results show that all four siRNA mixtures generated by RNase III induce 50\%-75\% decrease of the reporter activity at less than $10 \mathrm{nM}$ transfected concentration. In contrast, only one out of the five commercially available synthetic siRNAs showed comparable potency. These results suggest that one advantage of using enzymatic complex siRNA mixtures for RNAi is that, unlike single synthetic siRNAs, selecting a target region is not important to ensure potency.
\end{abstract}

\section{INTRODUCTION}

Once Fire and Mello described the phenomenon called RNA interference (RNAi) (1), several studies that revealed its molecular mechanisms followed (see References 2-5 for reviews). Several methods to apply RNAi in mammalian cells have been devised, including chemically synthesized small interfering RNAs (siRNAs) (6) and siRNA mixtures generated by in vitro enzymatic digestion $(7,8)$. Although guidelines and computational tools for designing effective siRNAs exist, experiments reveal that a majority of the synthetic siRNAs designed against a gene target are not very effective $(9,10)$. Many published siRNAs do not work at low concentrations and are commonly used at 100 $\mathrm{nM}$ or even higher $(9,11)$, despite the fact that off-target effects are observed at those concentrations (12-15).

In vitro processing of doublestranded RNA (dsRNA) by enzymatic digestion generates a population of siRNAs that targets multiple sites on the messenger RNA (mRNA). These have been shown to be more effective than single siRNA in silencing the target $(7,8)$. Although these results support the idea that effective siRNA mixtures are less subject to design rules than single siRNAs, we wanted to quantify differences in potency of different siRNA mixtures or siRNAs used at low concentrations.

Generally, RNAi efficiency is quantified either indirectly by assessing target protein concentrations by Western blot analysis or directly by measuring target mRNA levels by quantitative reverse transcription PCR (RT-PCR). Western blot analysis and quantitative RT-PCR have proven to be specific and accurate. However, they are costly in terms of experimental time, because assays have to be designed specifically for each new sample (i.e., quantitative PCR primers or antibodies). An alternative approach to assess knock-down potency is to link the target of RNAi to a reporter gene and quantify the knock-down effect by measuring the activity of the reporter. This principle has already been used successfully with intracel- lular fluorescent/luminescent reporters (16-20). Here, we applied this general approach using a secreted luciferase reporter gene.

The luciferase of Gaussia princeps (GLuc) has some unique properties among luciferases, such as high activity, stability, and the fact that it is efficiently secreted from mammalian cells by virtue of its native signal sequence $(21,22)$. In addition, its activity can be easily measured by available bioluminescent assays. The secretion of GLuc outside the cell facilitates the analyses of the samples. Since no cell lysis is required, rapid assays at different time points from the same experimental set are possible. We evaluated a GLuc-based reporter and used it to assay different synthetic siRNAs and different RNase IIIderived siRNA mixtures against the human $\mathrm{p} 53$.

\section{MATERIALS AND METHODS}

\section{pCMV-GLuc_p53 Reporter Vector}

A segment of the human 553 cDNA [National Center for Biotechnology Information (NCBI) accession no. NM 000546], from position 514 to 1581 , to be used as the target, was amplified by PCR and cloned. The primers used contain an XhoI restriction site as well as $5^{\prime}$ extensions compatible with the USER $^{\mathrm{TM}}$ technology for rapid cloning into linearized LITMUS ${ }^{\mathrm{TM}} \mathrm{U}$ plasmid using the USER enzyme system from New England Biolabs (Ipswich, MA, USA). The primer used were: $5^{\prime}$-GG AGACAUAAGCTTACTCGAG $C C C$ CCTCCTGGCCCCTGTCATCTT-3' (forward primer) and 5'-GGGAAAG UCTCGAGAAAGCTTGAGCCCCG GGACAAAGCAAATGGAA-3' (reverse primer) (USER ends are bold, XhoI sites are underlined, and specific p53 sequence is italic). The PCR fragment was first cloned into the LITMUS $\mathrm{U}$ vector. The resulting plasmid was digested by XhoI, and the p53 fragment was subsequently inserted in the $3^{\prime}$ untranslated region (UTR) of GLuc, at the XhoI site of the pCMVGLuc Control Plasmid (New England Biolabs) to produce the pCMV-GLuc_ p53 reporter vector. 


\section{p53 Transcription Templates for siRNA Mixtures}

A segment of human p53 (coordinates 553-1430) was inserted into the LITMUS 28i Vector (New England Biolabs). The insert was amplified with a T7 primer and digested with AciI. The resulting mixture of DNA fragments obtained was ligated at the Bst $\mathrm{BI}$ site of LITMUS 28i Vector. Individual clones containing p53 fragments were selected. The coverage of p53 sequence resulting from selected clones is shown in Figure 1B.

\section{Synthetic siRNAs and siRNA Mixture Preparations}

Synthetic siRNAs S1 (p53 pub. siRNA, no. 1024849) and S2 (Hs TP53_4_HP siRNA, no. SI00011662) were obtained from Qiagen (Valencia, CA, USA). S3 (Silencer $^{\circledR}$ validated siRNA, no. 42852), S4 (Silencer predesigned siRNA, no. 2625), and S5 (Silencer predesigned siRNA, no. 115183) were obtained from Ambion (Austin, TX, USA). Green fluorescent protein (GFP)-22 siRNA (80-11270) was obtained from Xeragon (now Qiagen), and Renilla luciferase (RL) siRNA (P-002070-01-20) was obtained from Dharmacon (Chicago, IL, USA). All synthetic oligonucleotides were prepared as $10 \mu \mathrm{M}$ solutions following the supplier protocols.

Mixtures of siRNAs targeting different regions of p53 (M2, M3, and M4) were generated from the transcription templates described above using the ShortCut ${ }^{\mathrm{TM}}$ RNAi kit (New England Biolabs) as follows. Transcription templates, flanked by opposing $\mathrm{T} 7$ promotors in LITMUS $28 \mathrm{i}$ Vector, were first amplified by PCR using T7 Minimal primers (New England Biolabs). PCR products were purified by ethanol precipitation by adding 1/10th volume of $3 \mathrm{M} \mathrm{NaOAc}$ and 2 volumes $100 \%$ ethanol. After 15 min incubation on ice, samples were centrifuged for $10 \mathrm{~min}$ at $16,000 \times \mathrm{g}$. Pellets were washed with 1 volume $80 \%$ ethanol and centrifuged $5 \mathrm{~min}$ at $16,000 \times g$. Transcription reactions were performed in $60 \mu \mathrm{L}$ in vitro transcription reagents and $0.5-2 \mu \mathrm{g}$ DNA template. Incubation of $4 \mathrm{~h}$ at

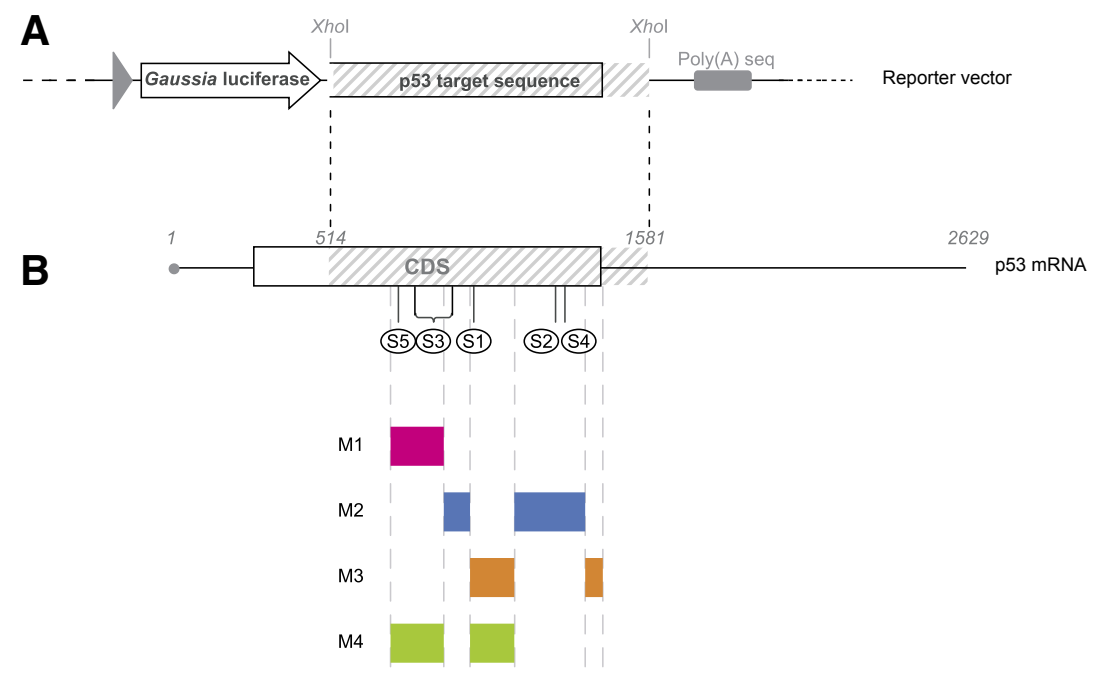

Figure 1. Gaussia princeps (GLuc) reporter and p53 regions targeted by the small interfering RNA (siRNA) reagents used. (A) Representation of the reporter vector with the p53 target sequence (hatched area) cloned in the 3' untranslated region (UTR) of GLuc gene downstream of the stop codon of GLuc. (B) Targeting regions of the specific synthetic siRNAs S1-S5 and the siRNA mixtures M1-M4, prepared as described, with the targeting region represented by colored bars (same color code as in Figure 3B). Mixtures M2-M4 target noncontiguous regions. The nucleotide targeting positions of the synthetic siRNAs are, respectively, for S1, 993; S2, 1275; S3, not specified (exon 6); S4, 1297; and S5, 751. The hatched area is the sequence used in the RNA interference (RNAi) reporter vector. mRNA, messenger RNA.

$42^{\circ} \mathrm{C}$ was followed by an annealing protocol of $72^{\circ} \mathrm{C}$ for $3 \mathrm{~min}, 68^{\circ} \mathrm{C}$ for $3 \mathrm{~min}, 65^{\circ} \mathrm{C}$ for $5 \mathrm{~min}, 62^{\circ} \mathrm{C}$ for $10 \mathrm{~min}$, and $42^{\circ} \mathrm{C}$ for $15 \mathrm{~min}$. Two $\mathrm{U}$ DNase I (New England Biolabs) per transcription reaction were added and incubated for $10 \mathrm{~min}$ at $37^{\circ} \mathrm{C}$. The suspension containing dsRNA was diluted 2-fold with water, in which then 1/10th volume of $3 \mathrm{M} \mathrm{NaOAc}$ was added and precipitated with 1 volume isopropanol. After 10 min of centrifugation at $16,000 \times g$, the pellet was washed with 1 volume $50 \%$ ethanol. After centrifuging for $5 \mathrm{~min}$ at $16,000 \times$ $g$, the supernatant was removed and the pellet allowed to air dry. The dsRNA was resuspended in the initial transcription volume of distilled water. Approximately $10 \mu \mathrm{g}$ each dsRNA were digested with RNase III to prepare heterogeneous mixtures of siRNA. Digestions were performed for $12 \mathrm{~min}$ at $37^{\circ} \mathrm{C}$ in $1 \times$ ShortCut reaction buffer, $20 \mathrm{mM} \mathrm{MnCl}{ }_{2}$, with a ratio of $2 \mathrm{U}$ enzyme ShortCut RNase III/ $\mu \mathrm{g}$ dsRNA. Reactions were terminated by adding $25 \mathrm{mM}$ EDTA. Digestion products were purified by ethanol precipitation, as described above, and resuspended in water. The M1 and CREB mixtures are commercially available, respectively, as p53 ShortCut siRNA mix and CREB
ShortCut siRNA mix (both from New England Biolabs).

To ensure using equal amounts of each siRNA reagent, we adjusted each siRNA concentration after comparison with three amounts of M1 $(1,5$, and 10 pmol) on 20\% TBE Gel (Invitrogen, Carlsbad, CA, USA) (see Figure 3A). Quantity One ${ }^{\circledR}$ software (Bio-Rad Laboratories, Hercules, CA, USA) was used for quantification after staining with GelStar ${ }^{\circledR}$ Nucleic Acid Stain (Cambrex, East Rutherford, NJ, USA).

\section{Cell Culture and Transfection}

HeLa cells and COS-7 cells were grown in Dulbecco's modified Eagle's medium (DMEM; GIBCO, Invitrogen) supplemented with $5 \%$ fetal bovine serum (FBS), $0.5 \times$ antibiotic-antimycotic solution $\left(\right.$ Cellgro $^{\circledR}$; Mediatech, Herndon, VA, USA). Twenty-four hours before transfection, the cells were split and plated into 96-well plates at 3000 cells/well. Cells were transfected in triplicate using TransPass ${ }^{\mathrm{TM}} \mathrm{R} 1$ transfection reagent (New England Biolabs) with the indicated amounts of siRNA, $100 \mathrm{ng}$ reporter plasmid $\mathrm{pCMV}$-GLuc_ $\mathrm{p} 53$, and $100 \mathrm{ng}$ constitutive $\beta$-galactosidase ( $\beta$-gal) expression vector when needed for normalization. 


\section{Quantitative RT-PCR}

The RNAqueous ${ }^{\circledR}$ kit (Ambion) was used to prepare total RNA from COS-7 cells. cDNA was made with the ProtoScript ${ }^{\circledR}$ First Strand cDNA Synthesis kit (New England Biolabs). Specific primers for quantitative PCR for p53 were designed and optimized (forward 5'-C CCCCTCCTGGCCCCTGTCATCTT $-3^{\prime}$ and reverse $5^{\prime}$-CGGGCGGGGGT GTGGAATCAAC- $3^{\prime}$ ) with an annealing temperature at $60^{\circ} \mathrm{C}$. The reactions were performed with $\mathrm{SYBR}^{\circledR}$ Green Supermix (Bio-Rad Laboratories). The p53 mRNA level was evaluated in each sample by relative quantitation with respect to $\beta$-actin using the $\Delta C_{T}$ method (www.gene-quantification.info) (23). Primers for $\beta$-actin were: forward $5^{\prime}$ TGCGTGACATTAAGGAGAAG-3' and reverse 5'-GCTCGTAGCTCT TCTCCA-3'. The p53 knock-down level was calculated as the ratio of p53 relative expression in p53 siRNAtreated cells to that in control siRNA- treated cells (CREB ShortCut siRNA mix) $24 \mathrm{~h}$ after transfection.

\section{Preparation of Cell Extracts and Western Blot Analyses}

COS-7 cells were lysed $48 \mathrm{~h}$ posttransfection on ice with $100 \mu \mathrm{L}$ lysis buffer ( $1 \%$ Triton ${ }^{\circledR} \mathrm{X}, 50 \mathrm{mM}$ Tris$\mathrm{HCl}, 150 \mathrm{mM} \mathrm{NaCl}$ ) for a 24-well plate, and supernatants containing protein were collected after centrifugation at $18,000 \times g$. For each sample, $10 \mu \mathrm{g}$ protein were resolved by Novex ${ }^{\circledR} 10 \%-20 \%$ Tris-glycine gel (Invitrogen) in sodium dodecyl sulfate (SDS) protein loading buffer (125 mM Tris, $1 \mathrm{M}$ glycine, $20 \mathrm{mM}$ SDS), and then electrotransferred to an Immobilon ${ }^{\circledR}-\mathrm{P}$ membrane (Millipore, Billerica, MA, USA). The membrane was blocked in 5\% milk, $1 \times$ Tris-buffered saline, $0.15 \%$ Tween $^{\circledR}$ 20 (TBST), and incubated overnight at $4^{\circ} \mathrm{C}$ with mouse monoclonal anti-p53 antibody (Cell Signaling Technology, Danvers, MA, USA) diluted 1:1000 in $\mathrm{TBST} / 5 \%$ bovine serum albumin

A

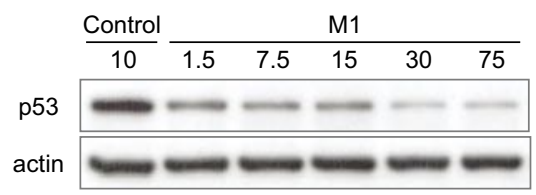

B

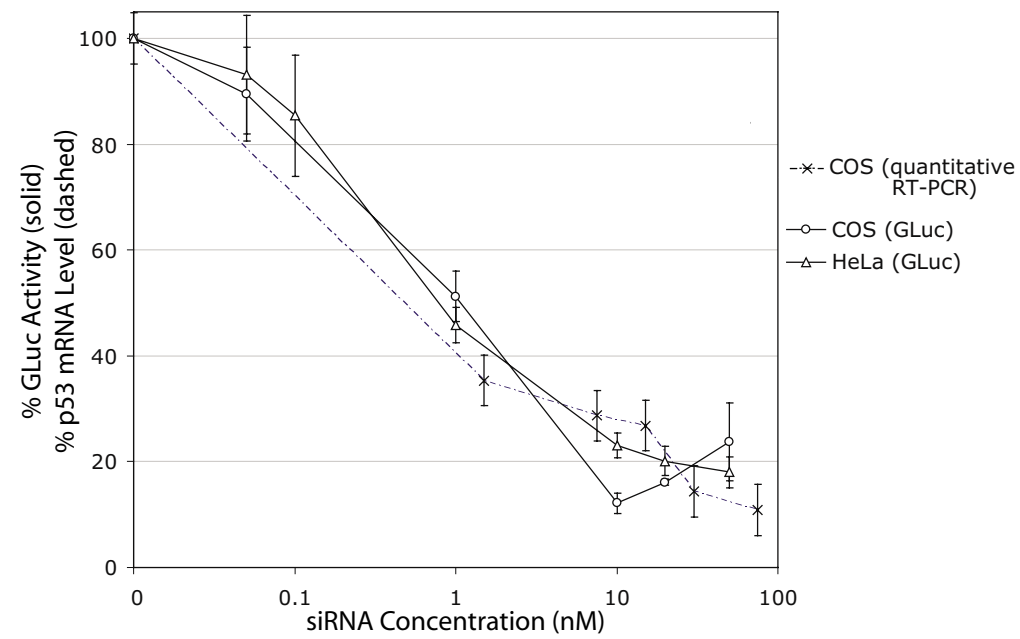

Figure 2. Gaussia princeps (GLuc) reporter validation by comparison to quantitative reverse transcription PCR (RT-PCR). (A) Western-blot analysis of the expression of the target p53 protein and the control actin protein $48 \mathrm{~h}$ after transfection in COS-7 cells of increasing concentrations of M1 small interfering RNA (siRNA) mixture. CREB ShortCut siRNA mix was used as negative control (control lane). (B) GLuc activity from supernatant of COS-7 and HeLa cells $24 \mathrm{~h}$ after transfection with pCMVGLuc_p53 and M1 siRNA mixture is plotted as a function of the transfected M1 siRNA concentration (solid lines). Error bars represent the standard deviation of triplicate measurements. This activity parallels the endogenous p53 messenger RNA (mRNA) level determined by quantitative RT-PCR (dashed line) using total RNA prepared from COS-7 cells transfected with the same M1 siRNA mixture.

(BSA), and rabbit polyclonal anti-actin (Sigma, St. Louis, MO, USA) diluted 1:3000 in TBST/5\% milk. Following incubation with horseradish peroxidase (HRP)-conjugated secondary antirabbit or anti-mouse diluted 1:2000 in TBST/5\% milk, signals were detected using the Phototope ${ }^{\circledR}$-HRP Western Blot Detection System (Cell Signaling Technology).

\section{Bioluminescent Assays}

For assaying GLuc activity, 20 $\mu \mathrm{L}$ each cell culture supernatant was transferred $24 \mathrm{~h}$ posttransfection into a black microtiter plate. Fifty microliters Gaussia luciferase assay working solution (New England Biolabs) were injected, and the activity was measured in relative luminescence units (RLU) with an integration time of $10 \mathrm{~s}$ after a 2-s delay.

After saving the supernatant for the GLuc assay, cells were lysed with $1 \times$ passive lysis buffer (Promega, Madison, WI, USA), and $40 \%$ of each sample was assayed for $\beta$-gal activity with the Galacto-Light ${ }^{\mathrm{TM}}$ system (Applied Biosystems, Foster City, CA, USA) with an integration time of $10 \mathrm{~s}$. All assays were performed with an $\mathrm{LMax}^{\mathrm{TM}}$ luminometer (Molecular Devices, Sunnyvale, CA, USA). Normalized knock-down was calculated from the ratio of GLuc/ $\beta$-gal to that obtained at each respective concentration of transfected GFP siRNA. Results obtained with the raw GLuc activity values showed exactly the same trends and lead to the same conclusions (data not shown).

\section{RESULTS AND DISCUSSION}

\section{Reporter Construction and Validation}

The pCMV-GLuc Control Vector, containing the constitutively expressed Gaussia luciferase, was used to construct the new reporter pCMVGLuc_p53, by cloning part of the p53 sequence downstream of the GLuc coding sequence stop codon (Figure 1A). Thus, the mRNA expressed from the plasmid contains both the reporter and the p53 target, which can be recognized and cleaved by the siRNARNA-induced silencing complex 
A

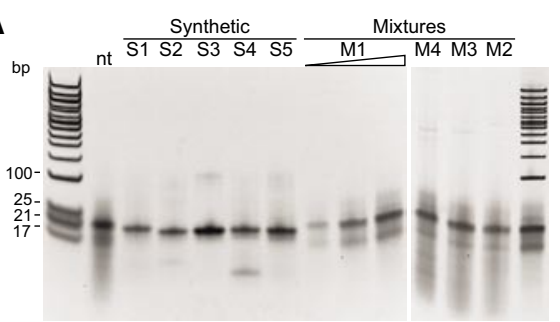

B

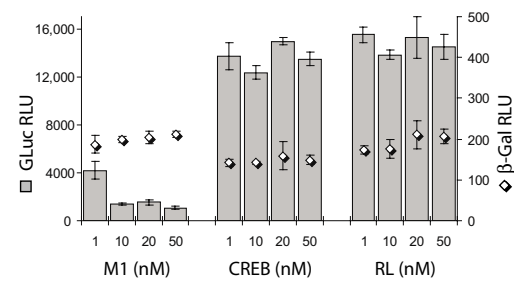

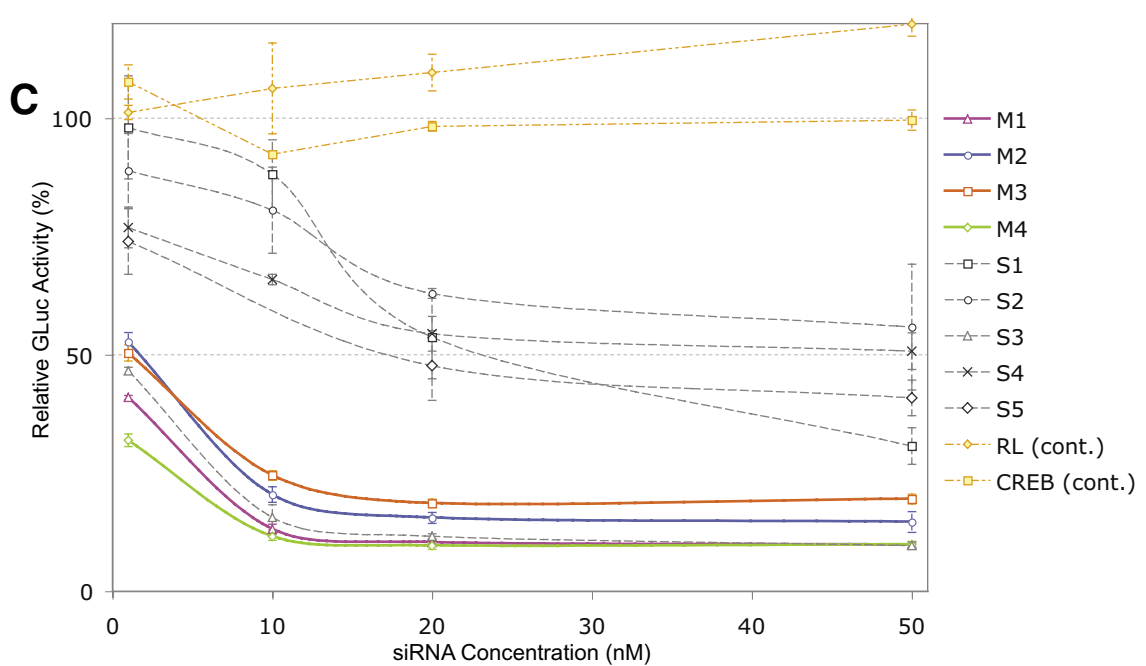

Figure 3. Concentration adjustment and silencing potency comparison of RNase III-generated small interfering RNA (siRNA) mixtures and synthetic siRNAs. (A) Polyacrylamide gel images used for concentration adjustment of the synthetic siRNAs and siRNA mixtures. Left panel, $5 \mu \mathrm{L}$ each synthetic siRNA was loaded next to 1,5 , and 10 pmol M1 mixture used as reference. The marker lane contains a mixture of $90 \mathrm{ng}$ siRNA marker and $100 \mathrm{ng}$ 100-bp DNA ladder (both from New England Biolabs). The lane marked nontargeting (nt) contains an unrelated siRNA mixture. Right panel, an equal volume of each siRNA mixture (M2-M4) was loaded next to a marker lane containing $100 \mathrm{ng}$ 100-bp DNA ladder and 10 pmol M1 siRNA mixture used as a reference. (B) Raw values of Gaussia luciferase (bars) and $\beta$-galactosidase ( $\beta$-gal; diamonds) activity, respectively, from supernatant and lysate of $\mathrm{HeLa}$ cells $24 \mathrm{~h}$ after cotransfection of pCMV-GLuc_p53 and different siRNA reagents. RLU, relative luminescence units. With the negative control (non-p53 targeting) siRNAs; synthetic siRNA (Renilla luciferase; RL) and siRNA mixture (CREB), the level of both Gaussia princeps (GLuc) and $\beta$-gal activities do not vary with increasing siRNA concentration up to $50 \mathrm{nM}$. With the M1 mixture targeting p53, there is a significant decrease of GLuc activity, while the level of $\beta$-gal does not change. (C) GLuc activity normalized as described herein, from culture supernatant of cells transfected with different concentrations of either synthetic siRNAs S1-S5 (gray dashed lines) or siRNA mixtures (color solid lines) is plotted as a function of transfected siRNA concentration. Targeted regions for mixtures M1-M4 and targeting positions for siRNAs S1-S5 are shown in Figure 1B (same color code). One synthetic and one mixture siRNA negative controls (yellow dashed lines) show no decrease of GLuc (or $\beta$-gal) activity with increasing transfected concentration.

(RISC) complex. The knock-down of the mRNA is reflected by decreased activity of the reporter.

The relative GLuc activity values obtained from HeLa or COS-7 cells transfected with different concentrations of M1 siRNA mixture was compared with the p53 mRNA levels measured by quantitative RT-PCR from similarly treated COS-7 cells. The comparison shows a very good correlation between the reporter assay and the knock-down results obtained by quantitative RT-PCR (Figure 2B). Western blot analyses with anti-p53

antibody show a substantial decrease of protein expression in proportion to the transfected siRNA concentration (Figure 2A). These results indicate that the reporter is functional and the measured activity is proportional to endogenous target mRNA levels caused by RNA silencing.

\section{Use of the Reporter for Comparisons of Synthetic siRNA and siRNA Mixtures Silencing Potency}

The Escherichia coli RNase III enzyme, in the presence of manganese, processes large dsRNA into a heterogeneous population of 21-bp siRNA appropriate for RNAi in mammalian cells (see Figure 3A). Enzymatically derived siRNA mixtures have the potential to include many individual siRNAs and theoretically should increase the chances of effective knock-down by targeting multiple sites on the mRNA. We tested this assumption in HeLa cells, using the secreted GLuc reporter to evaluate the effectiveness of siRNA mixtures generated by RNase III in comparison with different commercially available synthetic siRNAs against the same p53 target. The commercially available reagents included five synthetic siRNAs (S1-S5) and one RNase III-generated complex siRNA mixture (M1). In addition, we prepared three additional siRNA mixtures (M2-M4) targeting different regions of p53 by using a shotgun-cloning approach as described in the Materials and Methods section. The targeting region(s) on p53 for each reagent is indicated in Figure 1B. For the synthetic siRNAs, the position is provided by the manufacturer except in the case of $\mathrm{S} 3$, for which only the targeted exon is specified.

Each synthetic siRNA or siRNA mixture was transfected at concentrations ranging from 1 to $50 \mathrm{nM}$ along with the pCMV-GLuc_p53 reporter. GLuc activity was determined from culture supernatants as described in the Materials and Methods section. One synthetic siRNA against Renilla luciferase and one siRNA mixture against CREB were used as negative controls, which did not decrease the activity of either reporter, as opposed to M1 (Figure 3B). The silencing activities obtained from this study $24 \mathrm{~h}$ after transfection are shown in Figure 3C. The results show that all siRNA mixtures (M1-M4) achieved a 50\% knock-down at the lowest concentration used $(1 \mathrm{nM})$ and reached maximum knock-down $(75 \%-85 \%)$ of the reporter between 10 and $20 \mathrm{nM}$. In contrast, only one (S3) of the synthetic siRNAs showed comparable potency. Significantly, three out of five siRNAs tested (S1, S2, and S4) showed $<50 \%$ knock-down at 20 $\mathrm{nM}$, which is a maximum concentration recommended to avoid off-target effects (12-15). S2 and S4 had the lowest efficacy with knock-down reaching a 
plateau of $50 \%$ at $50 \mathrm{nM}$. These results show that the GLuc reporter we used was capable to quantify a wide range of silencing activities. Additionally, we conclude that complex siRNA mixtures, in general, have high potency, and unlike synthetic siRNAs, they do not seem to be dependent on target region specificity.

Different luciferase and GFP assay systems published previously also have shown a quantitative correlation between reporter and endogenous target silencing in agreement with our results using Gaussia luciferase (16-19). The GLuc reporter has two advantages: sensitivity and secretion. Because of the higher activity of the enzyme compared with firefly and Renilla luciferases (22), smaller scale assays are possible (e.g., 96- or 384-well format). In addition, because GLuc is secreted, samples do not require processing or lysis of cells before the assay, which permits a large number of samples to be assayed rapidly or multiple samplings over time. The assay is functional in standard tissue culture media (data not shown).

A recent paper suggested that testing three to five designed siRNAs may be necessary to ensure finding one that works, and that often, prediction programs are unreliable to the extent that some good siRNAs may be missed (10). Our results suggest that the use of siRNA mixtures overcomes the target site dependence restrictions imposed by single siRNAs $(9,10)$ by achieving a high knock-down efficiency at low concentration, as we found for all mixtures in our study. Although a precaution to be taken when choosing a template sequence for generating an siRNA mixture is to exclude regions of similarity with other genes, the use of programs such as DEQOR (24) facilitate the selection of the sequence.

Our study presents a rapid assay for siRNA functional validation and confirms that checking the potency of siRNA reagents is important before investing significant experimental time in RNAi knock-down experiments.

\section{ACKNOWLEDGMENTS}

This research was supported by New England Biolabs, Inc. We thank Dr.
Pierre-Olivier Estève for kindly providing the $\beta$-gal expression vector and Sarunna Jin for technical support. We also would like to thank Drs. Ana Egaña, Cathy Shea, and Barton Slatko for their helpful comments on the manuscript.

\section{COMPETING INTERESTS STATEMENT}

The authors are employees of New England Biolabs, Inc. (C.P. at the time of the work), which sells molecular biology reagents, including Gaussia luciferase products, and RNase IIIderived siRNA mixtures.

\section{REFERENCES}

1.Fire, A., S. Xu, M.K. Montgomery, S.A. Kostas, S.E. Driver, and C.C. Mello. 1998. Potent and specific genetic interference by double-stranded RNA in Caenorhabditis elegans. Nature 391:806-811.

2. Tomari, Y. and P.D. Zamore. 2005. Perspective: machines for RNAi. Genes Dev. 19:517-529.

3. Filipowicz, W., L. Jaskiewicz, F.A. Kolb, and R.S. Pillai. 2005. Post-transcriptional gene silencing by siRNAs and miRNAs. Curr. Opin. Struct. Biol. 15:331-341.

4.Hammond, S.M. 2005. Dicing and slicing: the core machinery of the RNA interference pathway. FEBS Lett. 579:5822-5829.

5. Valencia-Sanchez, M.A., J. Liu, G.J. Hannon, and R. Parker. 2006. Control of translation and mRNA degradation by miRNAs and siRNAs. Genes Dev. 20:515-524.

6. Elbashir, S.M., J. Harborth, W. Lendeckel, A. Yalcin, K. Weber, and T. Tuschl. 2001. Duplexes of 21-nucleotide RNAs mediate RNA interference in cultured mammalian cells. Nature 411:494-498.

7. Yang, D., F. Buchholz, Z. Huang, A. Goga, C.Y. Chen, F.M. Brodsky, and J.M. Bishop. 2002. Short RNA duplexes produced by hydrolysis with Escherichia coli Rnase III mediate effective RNA interference in mammalian cells. Proc. Natl. Acad. Sci. USA 99:99429947.

8. Kawasaki, H., E. Suyama, M. Iyo, and K. Taira. 2003. siRNAs generated by recombinant human Dicer induce specific and significant but target site-independent gene silencing in human cells. Nucleic Acids Res. 31:981-987.

9. Holen, T., M. Amarzguioui, M.T. Wiger, E. Babai, and H. Prydz. 2002. Positional effects of short interfering RNAs targeting the human coagulation trigger Tissue Factor. Nucleic Acids Res. 30:1757-1766.

10.Pei, Y. and T. Tuschl. 2006. On the art of identifying effective and specific siRNAs. Nat. Methods 3:670-676.

11.Shao, M.X.G. and J.A. Nadel. 2005. Dual oxidase 1-dependent MUC5AC mucin expression in cultured human airway epithelial cells. Proc. Natl. Acad. Sci. USA 102:767772.

12. Jackson, A.L., S.R. Bartz, J. Schelter, S.V. Kobayashi, J. Burchard, M. Mao, B. Li, G. Cavet, et al. 2003. Expression profiling reveals off-target gene regulation by RNAi. Nat. Biotechnol. 21:635-637.

13. Semizarov, D., P. Kroeger, and S. Fesik. 2004. RNA-mediated gene silencing: a global genome view. Nucleic Acids Res. 32:38363845 .

14. Persengiev, S.P., X. Zhu, and M.R. Green. 2004. Nonspecific, concentration-dependent stimulation and repression of mammalian gene expression by small interfering RNAs (siRNAs). RNA 10:12-18.

15. Fedorov, Y., E.M. Anderson, A. Birmingham, A. Reynolds, J. Karpilow, K. Robinson, D. Leake, W.S. Marshall, et al. 2006. Off-target effects by siRNA can induce toxic phenotype. RNA 12:1188-1196.

16. Yu, J.Y., S.L. DeRuiter, and D.L. Turner 2002. RNA interference by expression of short-interfering RNAs and hairpin RNAs in mammalian cells. Proc. Natl. Acad. Sci. USA 99:6047-6052

17. Kumar, R., D.S. Conklin, and V. Mittal. 2003. High-throughput selection of effective RNAi probes for gene silencing. Genome Res. 13:2333-2340.

18. Smart, N., P.J. Scambler, and P.R. Riley. 2005. A rapid and sensitive assay for quantification of siRNA efficiency and specificity. Biol. Proced. Online 7:1-7.

19. Rose, S.D., D.H. Kim, M. Amarzguioui, J.D. Heidel, M.A. Collingwood, M.E. Davis, J.J. Rossi, and M.A. Behlke. 2005. Functional polarity is introduced by Dicer processing of short substrate RNAs. Nucleic Acids Res. 33:4140-4156.

20.Sandy, P., A. Ventura, and T. Jacks. 2005. Mammalian RNAi: a practical guide. BioTechniques 39:215-224.

21. Verhaegent, M. and T.K. Christopoulos. 2002. Recombinant Gaussia luciferase. Overexpression, purification, and analytical application of a bioluminescent reporter for DNA hybridization. Anal. Chem. 74:43784385

22. Tannous, B.A., D.E. Kim, J.L. Fernandez, R. Weissleder, and X.O. Breakefield. 2005 Codon-optimized Gaussia luciferase cDNA for mammalian gene expression in culture and in vivo. Mol. Ther. 11:435-443.

23. Pfaffl, M.W. 2001. A new mathematical model for relative quantification in real-time RTPCR. Nucleic Acids Res 29:e45.

24. Henschel, A., F. Buchholz, and B. Habermann. 2004. DEQOR: a web-based tool for the design and quality control of siRNAs. Nucleic Acids Res. 32:113-120.

Received 15 November 2006; accepted 9 February 2007.

Address correspondence to George Tzertzinis, RNA Biology Division, New England Biolabs, Inc., 240 County Rd., Ipswich, MA 01938, USA. e-mail: tzertzin@neb.com

To purchase reprints of this article, contact: Reprints@BioTechniques.com 